\title{
Transplantation of autologous bone marrow pre-loaded ex vivo with oncolytic myxoma virus is efficacious against drug-resistant Vk*MYC mouse myeloma
}

\author{
Nancy Y. Villa ${ }^{1,2}$, Masmudur M. Rahman ${ }^{1}$, Joseph Mamola ${ }^{1}$, Meaghen E. Sharik $^{3}$, \\ Ana Lemos de Matos $^{1}$, Jacquelyn Kilbourne ${ }^{1}$, Kenneth Lowe ${ }^{1}$, Juliane Daggett- \\ Vondras $^{1}$, Julia D'Isabella ${ }^{1}$, Elizabeth Goras ${ }^{1}$, Marta Chesi ${ }^{3}$, P. Leif Bergsagel ${ }^{3}$ and \\ Grant McFadden ${ }^{1}$ \\ ${ }^{1}$ Biodesign Institute, Center for Immunotherapy, Vaccines and Virotherapy (CIVV), Arizona State University, Tempe, \\ AZ 85281, USA \\ ${ }^{2}$ Division of Hematology/Oncology, School of Medicine, Emory University, Atlanta, GA 32322, USA \\ ${ }^{3}$ Department of Medicine, Mayo Clinic, Scottsdale, AZ 85259, USA \\ Correspondence to: Grant McFadden, email: grantmcf@asu.edu \\ Keywords: myxoma virus; multiple myeloma; combination therapy; autologous transplantation; oncolytic virus \\ Received: December 18, $2021 \quad$ Accepted: February 14, $2022 \quad$ Published: March 03, 2022 \\ Copyright: (c) 2022 Villa et al. This is an open access article distributed under the terms of the Creative Commons Attribution License \\ (CC BY 3.0), which permits unrestricted use, distribution, and reproduction in any medium, provided the original author and source are credited.
}

\section{ABSTRACT}

Multiple myeloma (MM) is a hematological malignancy of plasma cells that remains incurable despite significant progress with myeloablative regimens and autologous stem cell transplantation for eligible patients and, more recently with $T$ cell redirected immunotherapy. Recently, we reported that ex vivo virotherapy with oncolytic myxoma virus (MYXV) improved MM-free survival in an autologoustransplant Balb/c mouse model. Here, we tested the Vk*MYC transplantable C57BL/6 mouse MM model that more closely recapitulates human disease. In vitro, the murine bortezomib-resistant Vk12598 cell line is fully susceptible to MYXV infection. In vivo results demonstrate: (i) autologous bone marrow (BM) leukocytes armed ex vivo with MYXV exhibit moderate therapeutic effects against MM cells pre-seeded into recipient mice; (ii) Cyclophosphamide in combination with BM/MYXV delays the onset of myeloma in mice seeded with Vk12598 cells; (iii) BM/MYXV synergizes with the Smac-mimetics LCL161 and with immune checkpoint inhibitor a-PD-1 to control the progression of established MM in vivo, resulting in significant improvement of survival rates and decreased of tumor burden; (iv) Survivor mice from (ii) and (iii), when re-challenged with fresh Vk12598 cells, developed acquired anti-MM immunity. These results highlight the utility of autologous BM grafts armed ex vivo with oncolytic MYXV alone or in combination with chemotherapy/immunotherapy to treat drug-resistant MM in vivo.

\section{INTRODUCTION}

Multiple myeloma (MM) is the second most common hematological malignancy in the world $[1,2]$. MM is characterized by the clonal expansion of malignant plasma cells (PCs) within a permissive bone marrow microenvironment that promotes the survival and proliferation of tumor cells [3-5]. Despite the remarkable progress in MM treatment, resulting in more prolonged disease-free survival, the disease remains incurable. Despite continued advances in new generations of drugs and novel therapies such as CAR-T cells, high dose ablative chemotherapy/radiotherapy along with autologous stem cell transplantation for eligible patients are still standard therapies used to treat myeloma patients [1]. However, the imminent relapse of the disease and its subsequent acquired resistance to existing drug therapies remain major challenges that still need to be overcome in order to assure long-term patient survival. 
Recently, we reported compelling experimental evidence indicating that the oncolytic myxoma virus (MYXV) can eliminate minimal residual MM disease in the setting of either allogeneic- [6] or autologous-stem [7] cell transplantation in an immunocompetent Balb/c mouse transplanted with mineral-oil induced plasmacytomas (MOPC)315.BM.dsRed cells [8]. Although the preclinical MOPC315.BM.dsRed myeloma model has been informative for us and others [8], and is well accepted as a preclinical model of MM, the MOPC model has some drawbacks. For example, in addition to the BM, the MM tumors can develop in the spleen and some mice exhibit extramedullary tumors (i.e., solid tumors outside the bone marrow compartment) [4]. Therefore, we aimed to overcome some of these limitations by using a mouse model of MM that more faithfully recapitulates the development, clinical manifestations and localization of the disease observed in human MM patients.

In 2008, Chesi and co-workers reported a murine C57BL/6-derived Vk*MYC MM preclinical model characterized by low proliferation of monoclonal plasma cells (PCs) within the Vk*MYC mice BM and secondary lymphoid organs, and therefore more closely resembling the feature of monoclonal gammopathy of undetermined significance (MGUS)/MM. Like in humans, this murine model produces abnormal high level of serum monoclonal Ig antibodies resulting in a detectable M-spike that can be measured in blood serum, and represents a clonal marker of tumor burden [9]. In terms of clinical manifestations, this model shows reduced levels of hemoglobin and bone mineral density (BMD), and MM-like kidney damage, which surrogates the clinical manifestation of the human disease [9]. Furthermore, this preclinical model has been used to accurately predict the chemotherapeutic effects of different anti-MM drugs [10]. Another peculiarity of the $\mathrm{Vk}^{*} \mathrm{MYC}$ model is the reproduction of cross talk between clonal PCs and the tumor microenvironment [10]. Upon harvesting serial passages of bortezomib (BOR)-resistant tumors from aged $\mathrm{Vk}^{*} \mathrm{MYC}$ mice, two independent BOR-resistant myeloma cell lines were generated and designated the transplantable BOR-resistant Vk12598 and the multidrug-resistant Vk12563 cells [10]. These cell lines can be transplanted to younger syngeneic C57BL/6 mice allowing for myeloma engraftment and aggressive disease development.

Here we demonstrated that murine BOR-resistant Vk12598 MM cells are fully susceptible to MYXV infection and oncolysis in vitro. On the other hand, in vivo data indicate that although virotherapy with free MYXV (i.e., un-armed vMyx-M135KO or armed human TNF expressing vMyx-hTNF recombinant constructs) delays the onset of MM disease, this monotherapy treatment was insufficient to eliminate or control the eventual progression of the disease. In contrast, transplantation with autologous BM cells ex vivo pre-loaded with either un-armed or TNF-armed
MYXV improved survival rates in a fraction of recipient mice pre-seeded with Vk12598 cells. Furthermore, combination therapy with the therapeutic alkylating agent cyclophosphamide (Cy), which, by itself, induces a partial response in patients with MM [11, 12] along with autologous BM ex vivo pre-loaded with either un-armed vMyx-M135KO, or vMyx-hTNF only delayed the onset of established MM disease inducing only a moderate improvement of survival rates that was not statistically significance as compared to single treatment with $\mathrm{Cy}$. In striking contrast, we found that treatment of recipient mice bearing Vk12598 cells with autologous bone marrow (BM) cells ex vivo treated with either un-armed or human TNF-armed MYXV virus constructs and in combination with the second mitochondrial-derived activator of caspases (Smac)-mimetics compound LCL161 and the immune checkpoint inhibitor (ICI) $\alpha$-PD-1 resulted in long-term survival and decrease of tumor burden in recipient mice. Importantly, when survivor mice from either of the last two cohorts were re-challenged with fresh Vk12598 cells, they were now resistant to the disease, suggesting that these mice had now acquired anti-myeloma immunity. Results from this study, demonstrate the therapeutic potential of using either un-armed or hTNF-armed MYXV in combination with auto-BM transplantation and chemo/ immunotherapy to control the progression of BORresistant MM disease.

\section{RESULTS}

\section{Murine Vk12598 MM cells are susceptible to MYXV binding and infection in vitro}

Similar to primary human myeloma cells, BORresistant Vk12598 myeloma cells are dependent on the native $\mathrm{BM}$ microenvironment to grow and proliferate [10]. Therefore, these murine Vk12598 cells cannot grow in vitro because of their very low proliferating index [10]. Although, we could not perform long-term in vitro experiments with these murine MM cells because of their imminent cell death in culture, we could perform shorttime in vitro experiments lasting $1 \mathrm{~h}$ or maximum $18 \mathrm{~h}$ at the most in order to determine the susceptibility of these BOR-resistant Vk12598 myeloma cells to MYXV binding and infection. In brief, $1 \times 10^{6} \mathrm{Vk} 12598$ cells isolated from $\mathrm{BM}$ or splenocytes derived from C57BL/6 mice pre-seeded for 4 weeks were used to determine virus binding or infection. For virus binding assays, fresh Vk12598 from either bone marrow (BM) or the spleen compartment were exposed to vMyx-M093L-Venus (a wild-type MYXV that expresses Venus-tagged M093 protein as virion component) at a multiplicity of infection (MOI) of 10 for $1 \mathrm{~h}$ at $4^{\circ} \mathrm{C}$, to allow virus binding to the cell surface. After $1 \mathrm{~h}$ the unbound virus was washed twice with cold 1x-PBS $+5 \%$ FBS. The levels of 
Venus-tagged virus binding to MM cells (i.e.,

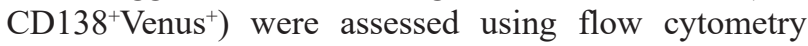
(Figure 1A). Also, to monitor active virus infection, fresh $1 \times 10^{6}$ Vk12598 cells were incubated with vMyx-GFP (MYXV that expresses GFP from a constitutive early/ late virus promoter) at $\mathrm{MOI}$ of 10 for $1 \mathrm{~h}$ at $37^{\circ} \mathrm{C}$, to allow virus adsorption. After this, cells were incubated overnight (i.e., $18 \mathrm{~h}$ ) at $37^{\circ} \mathrm{C}$ to allow virus entry and infection. Infection of $\mathrm{MM}$ cells (i.e., $\mathrm{CD} 138^{+} \mathrm{GFP}^{+}$) were determined using fluorescent microscopy and the levels of infection were assessed using flow cytometry (Figure 1B). Interestingly, loss in myeloma cell number was evident upon infection with vMyx-GFP for $18 \mathrm{~h}$, as compared to mock control (i.e., with mock: $41.8 \%$ vs. with MYXV: $5.2 \%$ viability), (Figure $1 \mathrm{C}$ ). The flow cytometry 2D-plots of a representative experiment are shown in the top panel of Figure 1C. Together, the data shown in Figure 1 demonstrate the susceptibility of BOR-resistant Vk12598 MM cells to MYXV binding and infection. Importantly, even when considering the short life span of these myeloma cells in culture, MYXV infection further abrogated the viability of the Vk12598 cells in vitro.

\section{Autologous bone marrow ex vivo loaded with unarmed-MYXV or TNF-armed MYXV delays the onset of MM disease in vivo and improves survival rates in mice pre-seeded with BOR- resistant murine Vk12598 myeloma cells}

Based on the in vitro results shown in Figure 1, we next tested whether virotherapy with MYXV alone or in combination with autologous stem cell transplantation (ASCT) can promote therapeutic effects in mice seeded with BOR-Vk12598 myeloma cells. We also wanted to exploit the therapeutic effects of an un-armed MYXV construct (using vMyx-M135KO) and a transgenearmed MYXV construct vMyx-hTNF (MYXV expressing human TNF) that has been recently shown to be more therapeutic than unarmed MYXV in a murine model of lung metastatic osteosarcoma [13]. To assess this, 8-weeks old immunocompetent recipient C57BL/6 mice were implanted with $1.0 \times 10^{6} \mathrm{Vk} 12598$ murine MM cells intravenously (i.v.) via the tail vein. One week after cancer implantation, mice cohorts were treated as follows: vehicle control (i.e., no BM transplant: PBS
A
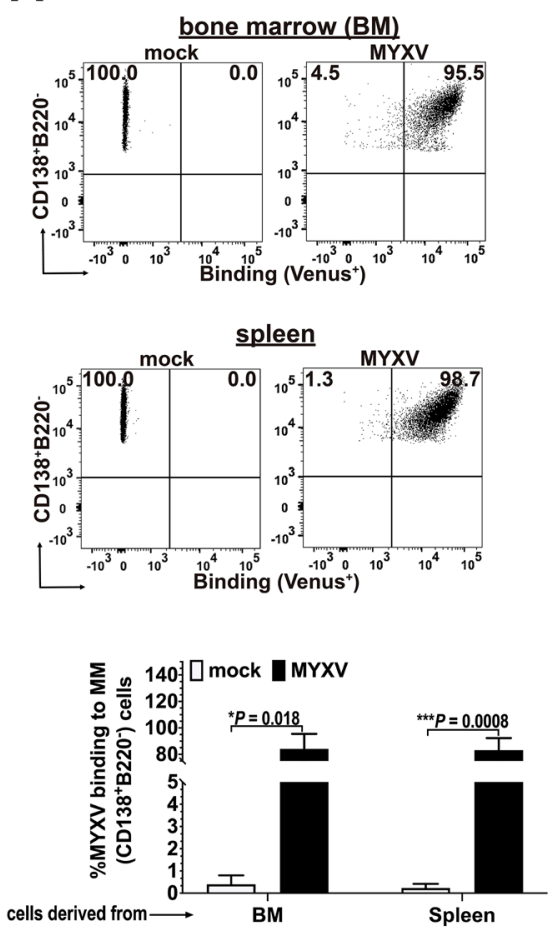

B
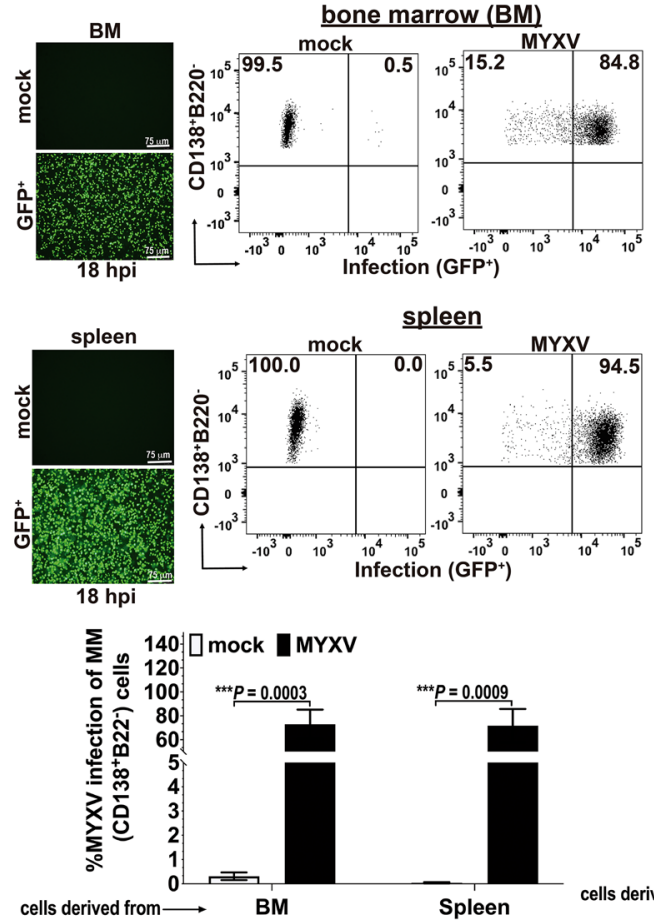

Virus Infection

spleen

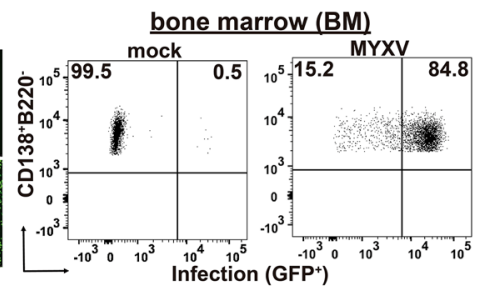

C MM Cell Viability Upon Virus Infection
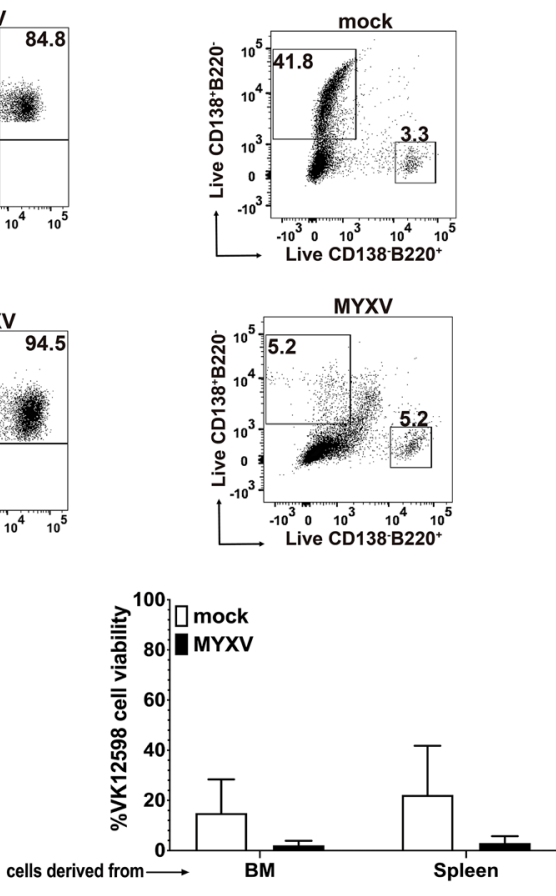

Figure 1: Murine BOR-resistant Vk12598 myeloma cells are susceptible to MYXV binding and infection in vitro. (A) Flow cytometry 2D-plots showing the levels of vMyx-M093L-Venus binding to fresh BOR-resistant Vk12598 MM cells derived from bone marrow and spleen (top and middle panels, respectively). The percentage of virus binding to Vk12598 cells derived from either BM, or spleen are summarized in (A)-bottom panel. (B), Fluorescent micrographs and flow cytometry 2D-plots showing the levels of infection of Vk12598 cells (top and middle panels). The percentages of infection (GFP expression from vMyx-GFP) of Vk12598 MM cells derived from $\mathrm{BM}$ or spleen after $18 \mathrm{~h}$ of incubation at $37^{\circ} \mathrm{C}$ is shown in bottom panel. (C) Flow cytometry 2D-plots of a representative experiment showing the percentage of MM cells remaining $\left(\mathrm{CD} 138^{+} \mathrm{B} 220^{-}\right)$upon mock-treatment or infection with vMyx-GFP after $18 \mathrm{~h}$ at $37^{\circ} \mathrm{C}($ top and middle panels, respectively). The percentages of MM cell viability after mock- or MYXV infection is shown in bottom panel. Plots represent the mean \pm standard deviation (SD) of at least 3 independent experiments. $P$ values are reported as statistically significant when ${ }^{*} P<0.05,{ }^{* * *} P 0.001$. 
only) ( $n=28$, cohort I); autologous C57BL/6 BM alone $(n=10$, cohort II); free un-armed vMyx-M135KO virus construct ( $n=10$, cohort III); autologous BM cells ex vivo treated with un-armed vMyx-M135KO virus construct ( $n=15$, cohort IV); free transgene-armed vMyx-hTNF virus construct ( $n=10$, cohort $\mathrm{V}$ ); autologous BM cells ex vivo treated with transgene-armed vMyx-hTNF virus construct ( $n=14$, cohort VI). All the test therapeutic treatments were delivered using the retro-orbital (r.o.) route. Recipient mice from each cohort received four consecutive treatments, each treatment every 3 days as described in Figure 2A. Figure 2B shows the KaplanMeier survival curves of these cohorts. The survival was scored at day 90 post-cancer implantation. Although the treatment with free MYXV (either armed or unarmed) slightly delayed the onset of the disease, the therapeutic effects were not statistically significant (i.e., percentage of survival: vehicle control $=0.0 \% ; \mathrm{BM}$ alone $=0.0 \%$, free $\mathrm{vMyx}-\mathrm{M} 135 \mathrm{KO}=10.0 \%$; free $\mathrm{vMyx}-\mathrm{hTNF}=$ $20.0 \%$ ). In contrast, mice treated with autologous BM ex vivo treated with unarmed vMyx-M135KO or armed vMyx-hTNF resulted not only in the delay of the onset of the disease but also some therapeutic effects, (i.e., percentage of survival: $\mathrm{BM}+\mathrm{vMyxM} 135 \mathrm{KO}=46.7 \%$; $\mathrm{BM}+\mathrm{vMyx}-\mathrm{hTNF}=50.0 \%$ ). However, the survival rates of these two cohorts were not statistically significant from each other, suggesting no functional difference between the un-armed virus vs. the hTNF-expressing armed MYXV construct. The results shown in Figure 2B summarize two independent in vivo experiments. In order to determine if cured mice developed acquired anti-tumor immunity to MM disease, survivor mice from our second in vivo experiment [i.e., survivor mice treated with either BM+vMyx-M135KO $(n=6)$, or $\mathrm{BM}+\mathrm{vMyx}-\mathrm{hTNF}(n=5)]$ were re-challenged with $1 \times$ $10^{6}$ fresh Vk12598 cells. Importantly, these latter mice survived for 212 days after re-challenging with no signs of myeloma disease as compared to mice bearing $\mathrm{MM}$ and treated with vehicle control (1x-PBS), (Figure 2C). Taking together, these data indicate that autologous BM ex vivo loaded with either un-armed MYXV (vMyxM135KO) or armed MYXV with human TNF (vMyxhTNF) had superior therapeutic effects than virotherapy with systemic infusion of either free unarmed or human TNF transgene armed MYXV.

\section{Combination of cyclophosphamide (Cy) with autologous bone marrow cells ex vivo loaded with MYXV delays the onset of Vk12598 multiple myeloma in vivo}

Because our goal was to explore different therapeutic strategies that effectively target and eliminate resistant MM cells in vivo, we decided to exploit combination therapy with the alkylating agent cyclophosphamide (Cy) along with auto-transplantation of BM ex vivo treated with un-armed or armed MYXV. Cy is one of the upfront chemotherapeutic agents used to treat patients with MM $[11,12]$. Cy has been reported to potentiate $\mathrm{T}$ cell redirected therapy and other adaptive immune responses against established tumors by abrogating regulatory $\mathrm{T}$ cells and restores T and NK cell functions [14-18]. In addition to this, $\mathrm{Cy}$ induces an acute secretory activating phenotype that promotes non-differentiated macrophage (MФ) infiltration and phagocytosis of tumor cells [12]. Because Cy partially abrogates the progression of MM resulting in tumor reduction $[11,12]$, we hypothesized that $\mathrm{Cy}$ might synergize with autologous BM preloaded with MYXV to improve survival of mice bearing established BOR-resistant MM disease. In order to test this hypothesis, 8-weeks old immunocompetent C57BL/6 recipients were injected with $1.0 \times 10^{6} \mathrm{Vk} 12598 \mathrm{MM}$ cells intravenously (i.v.) via the tail vein. Unlike the previous study (Figure 2), in this study myeloma cells were allowed to establish later-stage disease for 3 weeks prior to initiation of therapy as described in Figure 3A. After this, mice were treated with either vehicle control (i.e., 1x-PBS) ( $n=17$, cohort I) via the retro-orbital (r.o.) route, or with $100 \mathrm{mg} / \mathrm{Kg}$ Cy alone delivered via the intra-peritoneally (i.p.) route, twice, each dose one week apart (i.e., at days 1 and 7$),(n=12$, Cohort II) in order to induce minimal residual disease (MRD) (Figure 3A). At day 14 after drug treatment, some of the mice that were pretreated with 100 $\mathrm{mg} / \mathrm{Kg}$ of $\mathrm{Cy}$ were transplanted with either $\mathrm{BM}$ ex vivo pre-loaded with un-armed vMyx-M135KO $(n=8$, cohort III), or with BM ex vivo pre-loaded with armed vMyxhTNF ( $n=9$, cohort IV). Treatments with BM ex vivo preloaded with MYXV were delivered using the retro-orbital (ro) route. The survival curves of these cohorts are shown in Figure 3B. As can be seen, the treatment with Cy alone, or in combination with autologous BM/MYXV resulted in some improvement of survival rates of mice seeded with $\mathrm{MM}$ as compared with mice treated with vehicle control (i.e., percentage of survival with vehicle control $=0.0 \% ; \mathrm{Cy}$ alone $=25.0 \% ; \mathrm{Cy}+\mathrm{BM} / \mathrm{vMyx}-\mathrm{hTNF}=$ $22.0 \% ; \mathrm{Cy}+\mathrm{BM} / \mathrm{vMyxM} 135 \mathrm{KO}=50.0 \%$ ). However, we could not establish statistical differences among cohorts II, III and IV. We speculate that in vivo pretreatment of myeloma cells with Cy can compromise MYXV spread or distribution within the tumor milieu, which ultimately hampers viral oncolysis specifically at high MOIs (i.e., MOI $=10$ ). In fact, it has been reported by others that Cy in combination with low doses of rQNesting34.5, a modified herpes simplex virus-1 (HSV-1), increased survival of established xenograft glioma, as compared to combination of $\mathrm{Cy}$ and high doses of rQNesting34.5 [19]. Another possibility is that $\mathrm{Cy}$ in combination with high load levels of virus might be very toxic, limiting the therapeutic effects of this combination treatment (i.e., Cy and BM/MYXV) [19]. In addition to this, it is known that in terms of innate immune mechanisms, $\mathrm{Cy}$ stimulates antiviral immune defenses. This latter could 
result in increased antibody-mediated viral neutralization and ultimately clearing the oncolytic virus [20]. Taken together, the results shown in Figure 3B demonstrate that combination therapy with the alkylating agent $\mathrm{Cy}$ plus autologous BM transplant ex-vivo pre-loaded with MYXV delays the progression of BOR-resistant Vk12598 MM cells. However, we could not establish significant therapeutic benefits in terms of improved survival rates

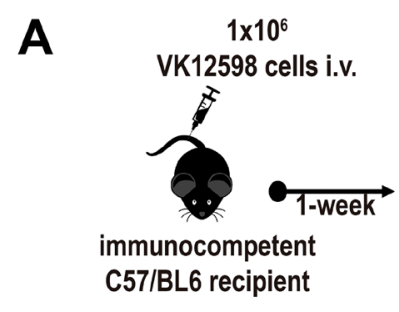

B

\section{Cohorts}

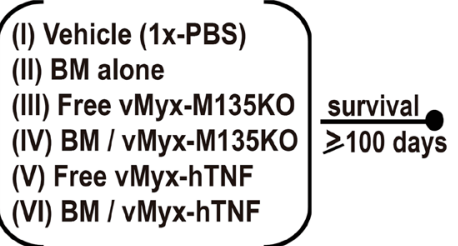

4 independent treatments: each treatment every 3 days
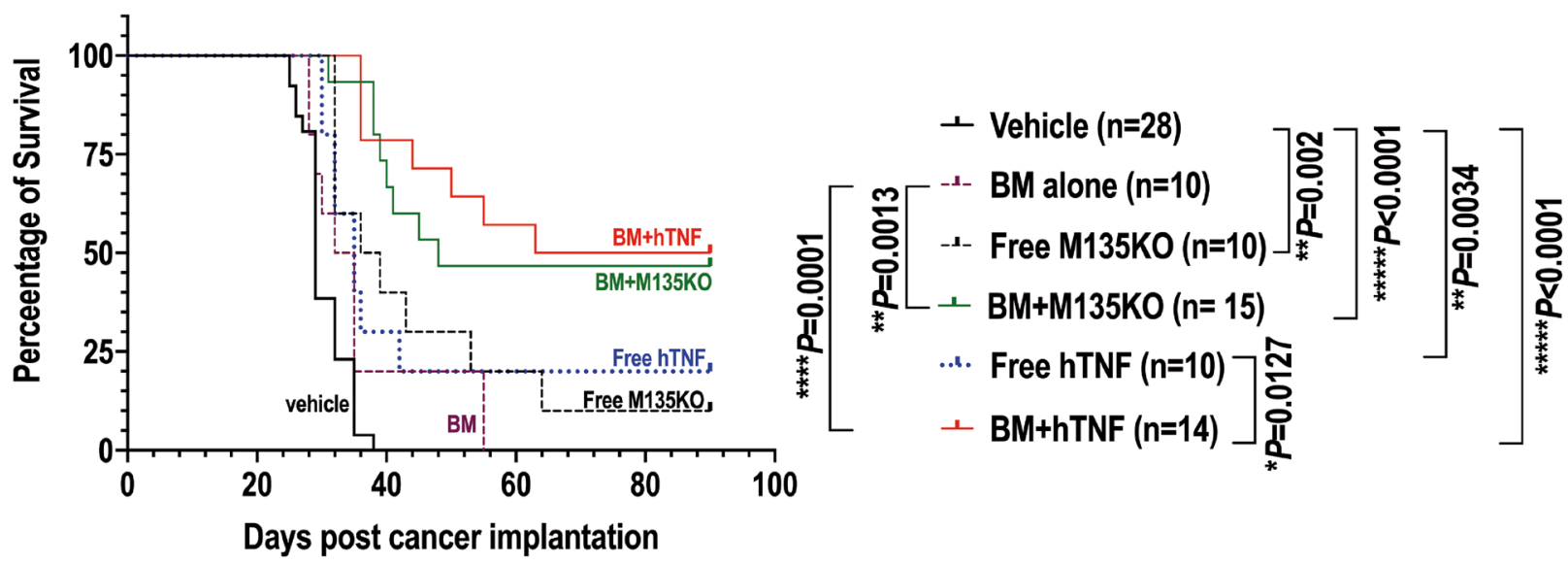

C

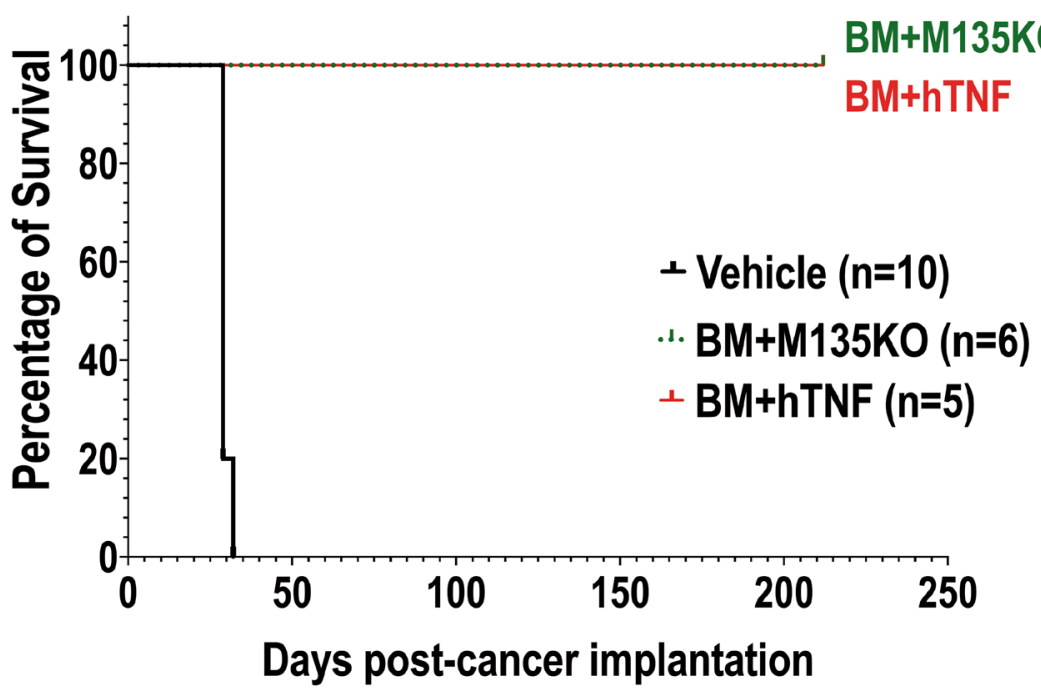

Figure 2: Autologous bone marrow ex vivo loaded with MYXV not only resulted in improvement of survival rates of recipient mice pre-seeded with Vk12598 myeloma cells, but also a fraction of these survivor mice became resistant after re-challenging them with myeloma cells. (A) Diagram describing the experimental design including the treatment time-line after myeloma implantation. (B) Survival curves at day 90 post-cancer implantation, of different cohorts comparing the therapeutic effects of virotherapy with free un-armed vMyx-M135KO virus alone or in combination with BM transplantation versus free armed vMyx-hTNF virus alone or in combination with auto-BM transplantation. (C) Survival curves of survivor mice re-challenged with fresh Vk12598 multiple myeloma cells. In panel (C), cohort treated with 1x-PBS was used as a control to demonstrate the susceptibility of the mice seeded with fresh Vk12598 MM cells. The $P$ values for log-rank Mantel-Cox survival test, are shown in (B). $P$ values are reported as statistically significant when ${ }^{* *} P 0.01,{ }^{* * *} P 0.001,{ }^{* * * *} P \leq 0.0001$. 
upon treatment of the recipient mice with $\mathrm{Cy}$ alone or $\mathrm{Cy}$ in combination with $\mathrm{BM} / \mathrm{M} 135 \mathrm{KO}$, or $\mathrm{BM} / \mathrm{hTNF}$.

\section{Autologous murine bone marrow cells ex vivo loaded with MYXV synergizes with the Smac- mimetics compound LCL161 and the checkpoint inhibitor $\alpha$-PD-1 against murine BOR-resistant Vk12598 multiple myeloma cells}

Results shown in Figure 3B suggest that MYXV can be used as adjunct therapy to treat aggressive and BOR-resistant Vk12598 MM cells in vivo. Knowing this, we next explored combination therapy with the Smacmimetics compound LCL161 along with immunotherapy using the immune checkpoint inhibitor (ICI) $\alpha$-PD-1. Smac-mimetics LCL161 compound has been developed as an antagonist of the cellular inhibitor of apoptosis cIAP-1 and -2 and has been unsuccessfully evaluated in clinical trials for its ability of induce TNF-mediated apoptosis of cancer cells $[12,21]$. On the other hand, by activating the non-canonical NFkB pathway, LCL161 has been shown to manipulate the tumor microenvironment inducing immune activation and resulting in long-lasting protection against myeloma progression in vivo [12]. Furthermore, combination therapy with LCL161 plus $\alpha-P D-1$ strikingly improved survival rates of recipient mice bearing MM [12]. Based on these reported results, we decided to investigate sequential treatments with unarmed MYXV or armed MYXV with the hTNF transgene alone or in combination with autologous BM transplant and with LCL161 $+\alpha$-PD-1 against MM. In brief, eightweeks old C57BL/6 mice were injected with $1.0 \times 10^{6}$ Vk12598 myeloma cells intravenously (i.v.) via the tail vein. One week after cancer implantation, mice were treated on day 1, 4, 8 and 11 with: $50 \mathrm{mg} / \mathrm{kg}$ of LCL161 via the oral gavage (o.g.) route or $10 \mathrm{mg} / \mathrm{mL}$ of $\alpha$-PD-1 intraperitoneally (i.p.), or both LCL161 $+\alpha$-PD-1. On days 2, 5, 9 and 12, these mice were injected with autologous free viruses or with $\mathrm{BM}$ ex vivo pre-loaded with either
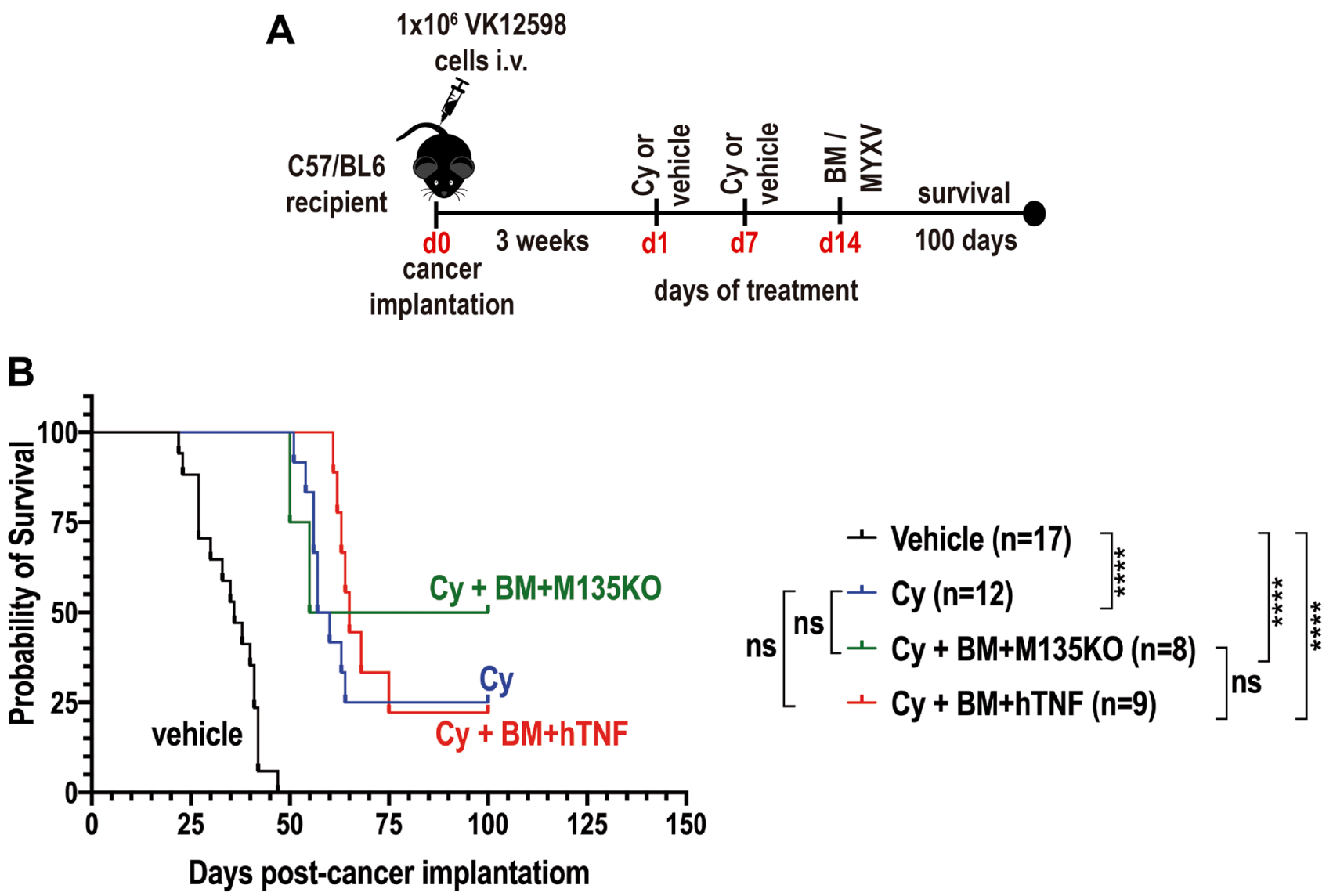

Figure 3: Combination therapy with cyclophosphamide (Cy) and autologous bone marrow (BM) pre-loaded with MYXV delay the onset of MM. (A) Diagram describing the experimental design including the treatment time-line after myeloma implantation. (B) Survival curves of different cohorts comparing the therapeutic effects of $100 \mathrm{mg} / \mathrm{Kg}$ cyclophosphamide (Cy) alone versus Cy followed by transplant with autologous BM pre-loaded with un-armed vMyxM135KO virus versus Cy followed by transplant with autologous BM pre-loaded with armed vMyx-hTNF virus. (B) Survival curves show the number of treated mice (n), survival (in days) from the beginning of cancer implantation, and $P$ values for log-rank Mantel-Cox survival test. $P$ values are reported as not statistically significant (ns) when $P>0.05$ or statistically significant when ${ }^{* * * *} P \leq 0.0001$. 
un-armed vMyx-M135KO or with armed vMyx-hTNF via the retro-orbital (r.o.) route. Control mice were treated only with vehicle. Under these experimental conditions, we found that combination of LCL161 $+\alpha$-PD-1 coupled with autologous BM pre-loaded with either vMyx-M135KO or with vMyx-hTNF significantly improved survival rates of mice bearing Vk12598 myeloma cells as compared with the combination of LCL161 $+\alpha$-PD-1 with either free vMyx-M135KO or vMyx-hTNF (i.e., percentages of survival upon combination therapy with LCL161 $+\alpha-\mathrm{PD}-$ 1 followed by: BM/vMyx-M135KO $=75.0 \%$; BM/hTNF $=60.0 \%$; free vMyx-M135KO $=20.0 \%$; free vMyx$\mathrm{hTNF}=20.0 \%$; LCL161 $+\alpha-\mathrm{PD}-1=26.7 \%$ ). Under these experimental conditions we did not observe increased survival when recipient mice were treated with LCL161 $+\alpha$-PD-1 only as reported in a former publication using transplantable murine $\mathrm{Vk}^{*} \mathrm{MYC}$ model [12], Figure 4B. This discrepancy might be attributed to the origin of the mice (Charles River in this case) and/or to variations in the diet and consequently the microbiome of our experimental mice. In terms of tumor burden, we also measured the levels of M-Spike from recipient's serum at the indicated time points, which did show significant tumor reduction by day 25 in the LCL161 $+\alpha-$ PD- 1 cohort but by day 55 , the MYXV-treated mice consistently showed further reductions in tumor burden (Figure 4C).

\section{Therapy with autologous BM ex vivo pre-loaded with MYXV and in combination with LCL161 and $\alpha$-PD-1 was curative for a fraction of survivor mice that were re-challenged with fresh murine Vk12598 myeloma cells}

The in vivo results shown in Figure 4B, clearly reveal greater therapeutic effects when recipient mice seeded with Vk12598 cells were treated with autologous BM loaded with MYXV (i.e., vMyx-M135KO or vMyx-hTNF) in combination with the Smac-mimetics compound LCL161 plus $\alpha-\mathrm{PD}-1$. Therefore, in order to determine whether or not these combination treatments can be curative for those survivor mice, $1 \times 10^{6} \mathrm{Vk} 12598$ cells, were implanted intravenously (i.v.) via the tail vein, in these survivor mice from the combinatorial treatment cohorts. Importantly, we found that $75 \%$ of those mice treated with LCL161+ $\alpha$-PD-1 followed by $\mathrm{BM} / \mathrm{vMyx}-\mathrm{M} 135 \mathrm{KO}$ and $50 \%$ of those mice treated with LCL161+ $\alpha$-PD-1 followed by BM/vMyx-hTNF survived for 245 days after re-challenging with murine Vk12598 cells (Figure 5). Furthermore, we found that mice that were first treated with LCL161+ $\alpha$-PD-1 followed by free $\mathrm{M} 135 \mathrm{KO}$ or hTNF-armed MYXV eventually succumbed when re-challenged with fresh Vk12598 (data not shown). Interestingly, $50 \%$ of survivor mice $(n=4)$ that were originally treated LCL161+ $\alpha$-PD1 tolerated re-challenge with the same myeloma cells (Figure 5A), which is in agreement with data reported by Chesi and co-workers [12]. The levels of M-spikes shown in Figure 5B, correlates with the survival rates of four different cohorts. Together, our data support the notion that combination therapy with LCL161+ $\alpha$-PD-1 with $\mathrm{BM}$ ex vivo loaded with MYXV induces resistance to secondary challenge with Vk12598 cells.

\section{DISCUSSION}

Treatment of MM is challenging because the disease inevitably relapses and becomes resistant to current standard therapies. Oncolytic viruses (OVs) have recently emerged as a potential therapeutic alternative to treat hematological malignancies like $\mathrm{MM}$ in preclinical models and/or clinical trials [22-26]. Therapeutic OVs can be used alone or as an adjunct therapy in combination with standard chemotherapeutic agents [22]. Depending on the types of tumors and their localization, OVs can be delivered intratumorally, systemically as free virus or after ex vivo loading onto carrier cells. Each of these delivery methods can impact the therapeutic outcomes. The systemic delivery of any free OV faces several therapeutic challenges including: (i) neutralization by circulating serum factors such as antibodies and complement, (ii) sequestration in secondary lymphoid organs like the spleen, or clearance in the liver, (iii) often targeting to vascular endothelial cells [27]. In contrast, using autologous BM leukocytes or PBMCs as carrier cells to deliver OVs to tumor sites has several advantages over the systemic delivery of free virus to selectively reach the tumor microenvironment. In the past, our lab has demonstrated that allogeneic or autologous BM grafts pre-loaded ex vivo with oncolytic MYXV can circumvent some of the disadvantages associated with the systemic delivery of free virus $[6,7,13]$.

Another important advantage of using OVs is that they can activate the innate or adaptive immune system on their own or when combined with chemotherapeutic agents and/or ICIs [28, 29]. Furthermore, OVs can be genetically engineered to allow expression of therapeutic transgenes directly in the tumor bed to change the tumor microenvironment (TME) and to recruit anti-tumor immune cells. We recently demonstrated that autologous PBMCs ex vivo loaded with an engineered oncolytic MYXV expressing human TNF (vMyv-hTNF) was an effective therapeutic strategy against murine K7M2 osteosarcoma cells in a lung metastatic preclinical syngeneic murine model, whereas unarmed MYXV was ineffective [13].

In this study, we used the $\mathrm{Vk}^{*} \mathrm{MYC}$ MM model because it faithfully recapitulates the localization of the myeloma disease within the bone marrow as well as the clinical manifestation of the disease including bone damage (paralysis), renal failure [9, 12]. In addition to this, the Vk*MYC model allows determination of tumor burden (i.e., levels of circulating M-Spike protein) in 
A

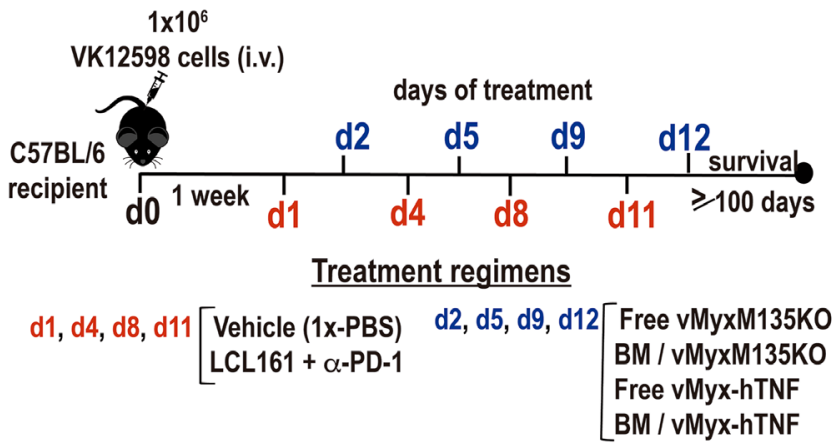

B
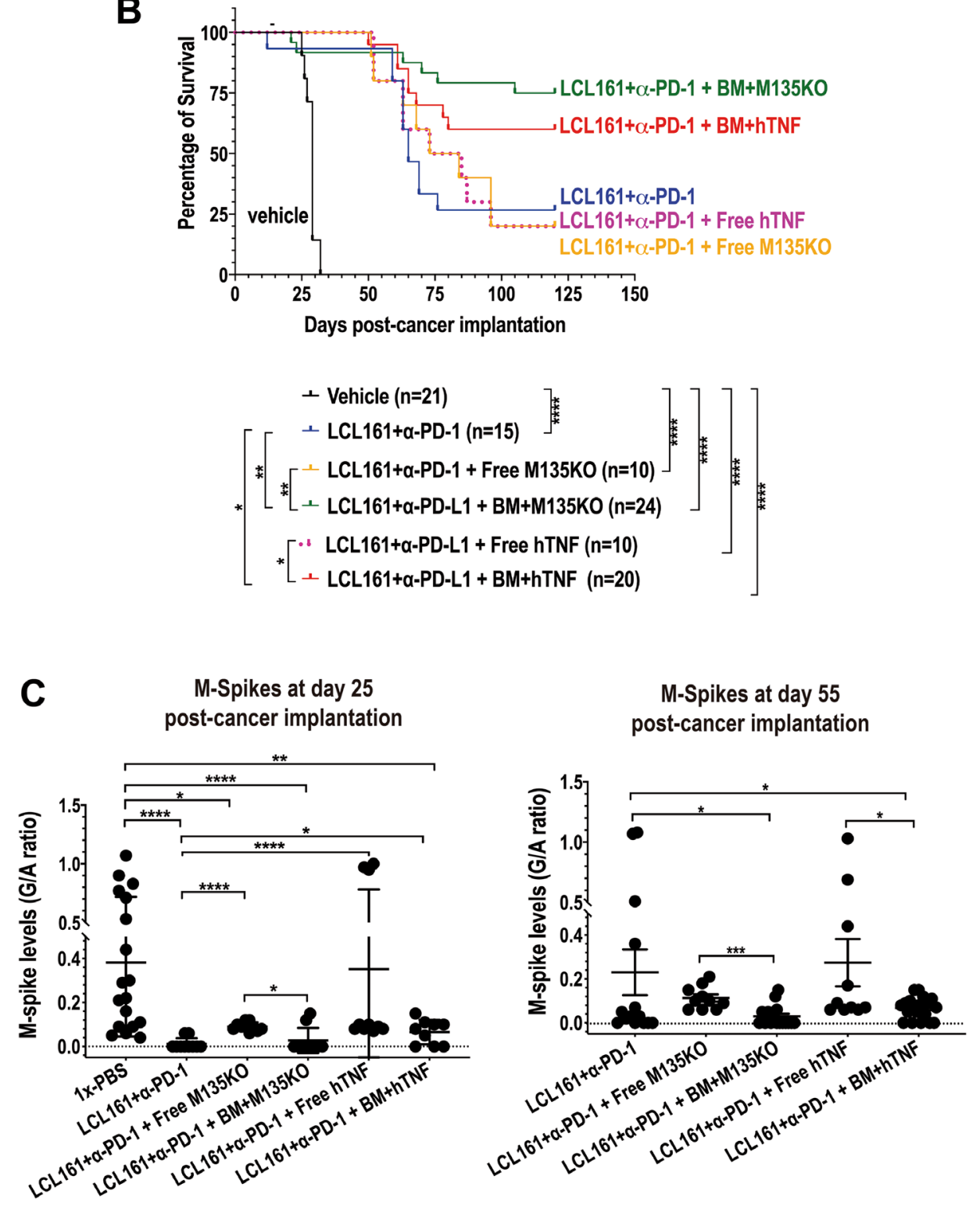

Figure 4: Autologous BM ex vivo preloaded with either un-armed MYXV, or with hTNF transgene armed MYXV synergizes with LCL161 + $\alpha$-PD-1 against BOR-resistant Vk12598 myeloma cells in vivo. (A) Diagram describing the experimental design including the treatment time-line after myeloma implantation. (B) Survival curves of mice treated with LCL161 + $\alpha$-PD-1 +/- free vMyxM135KO versus free vMyx-hTNF, LCL161 + $\alpha$-PD-1 + BM ex vivo pre-loaded with either vMyxM135KO, or vMyxhTNF. Survival curves (B) show the number of treated mice (n), survival (in days) from the beginning of cancer implantation, and $P$ values for log-rank Mantel-Cox survival test. $P$ values are reported as not statistically significant (ns) when $P>0.05$ or statistically significant when ${ }^{*} P<0.05,{ }^{* *} P 0.01,{ }^{* * *} P 0.001,{ }^{* * * *} P \leq 0.0001$. (C) Plots the M-spike levels in sera in response to each indicated treatment regimen at day 25 or at day 55. Student's $t$-test was used to compare the mean of each M-spike with every other M-spike mean post cancer implantation. 


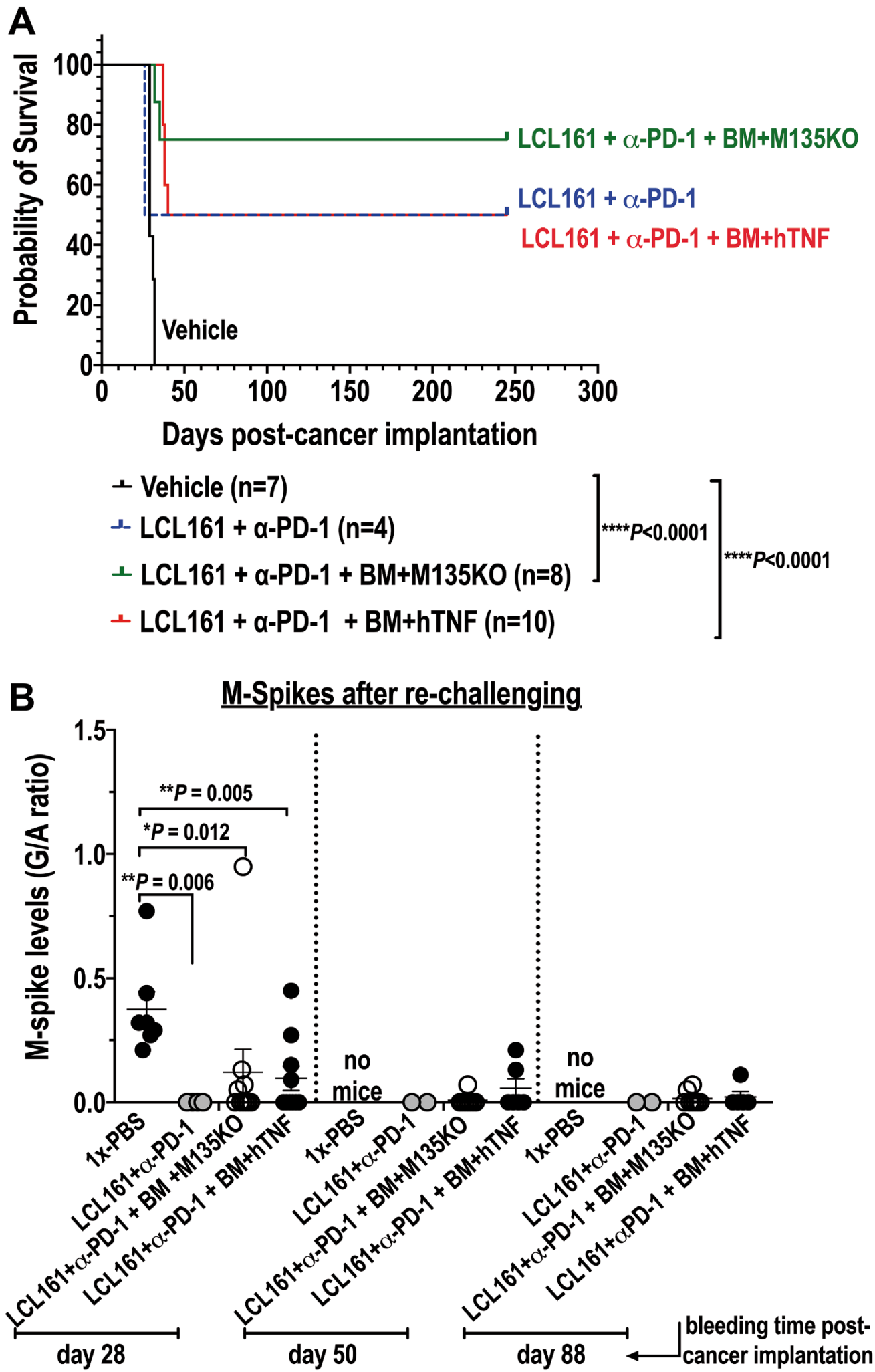

Figure 5: Combination therapy with auto-BM ex vivo loaded with either un-armed or hTNF armed MYXV with LCL161 and $\alpha$-PD-1curative to mice seeded with BOR-resistant Vk12598 cells. (A) Shows survival curves of survivor mice re-challenged with Vk12598 multiple myeloma cells. (B) shows tumor burden (levels of M-spike) of those mice re-challenged with the Vk12598 myeloma cells. Survival curves (A) show the number of treated mice, survival (in days) from the beginning of re-challenging, and $P$ values for log-rank Mantel-Cox survival test. Unpaired two-tailed $t$ test $P$ values are reported in (B). $P$ values are reported as not statistically significant (ns) when $P>0.05$ or statistically significant when ${ }^{*} P<0.05,{ }^{* *} P 0.01,{ }^{* * *} P 0.001,{ }^{* * * *} P \leq 0.0001$. No mice means that the mice at that particular time point have already succumbed to the disease. 
the mouse serum using serum protein electrophoresis (SPEP) assay, which is routinely used in the clinic to track MM development and progression. Here we set out to investigate and compare the therapeutic effects of virotherapy with un-armed MYXV (i.e., vMyX-M135KO) with a recombinant MYXV armed with the human TNF (i.e., vMyx-hTNF), and also compare the anti-cancer efficacy of delivering virus systemically via retro-orbital (r.o.) injection of naked virus versus autologous BM ex vivo loaded with each of the above MYXV constructs alone or in combination with chemo/immunotherapy using the transplantable murine $\mathrm{Vk}^{*} \mathrm{MYC}$ multiple myeloma model.

Our in vitro studies demonstrate the capacity of MYXV to bind BOR-resistant Vk12598 multiple myeloma cells. Furthermore, we were able to show that MYXV can also infect these MM cells (Figure 1). Being able to access the levels of MYXV binding and virus infection of these MM cells is relevant taking in consideration that Vk12598 cells cannot be cultured for long periods of time in vitro. The fact that MYXV can infect and compromise the viability of these myeloma cells in a short period of time is relevant and suggest the potent oncolytic activity of MYXV against these BOR-resistant MM cells.

To determine if our in vitro results can be recapitulated in vivo, bortezomib-resistant Vk12598 MM cells were seeded in immunocompetent C57BL/6 mice and then transplanted with auto-BM cells that had been ex vivo loaded with either unarmed vMyx-M135KO or transgenearmed vMyx-hTNF virus constructs. Our in vivo results demonstrated that under these therapeutic conditions, not only was the onset of the disease delayed, but also, overall survival rates increased in a fraction of immunocompetent C57BL/6 mice pre-seeded with Vk12598 myeloma cells as shown in Figure 2B. However, we could not establish statistically significant differences upon transplantation with auto-BM ex vivo loaded with either of the described virus constructs, suggesting the therapeutic effects emanate from the virus backbone, not the TNF transgene tested. These in vivo results although were unpredicted based on our in vitro results and the fact that we had expected higher therapeutic effects of the armed MYXV construct with a pro-inflammatory cytokine like TNF, as had been observed in a syngeneic lung metastatic osteosarcoma model [13]; however our in vivo results were not entirely surprising since TNF has been reported by others to promote IL-6, a propagating factor for MM $[30,31]$ that suppresses apoptosis of MM by activating the NF- $\kappa \mathrm{B}-$ pathway [32]. This is in contrast of recently published meta-analysis demonstrating the protective effect of TNF against MM risk in the dominant model and allele analysis [33]. These published inconsistences in regards the effects of TNF on myeloma progression or inhibition prompted us to investigate the therapeutic effects of this cytokine in the context of oncolytic virotherapy with MYXV. Taken together, we observed some therapeutic effects in mice seeded with MM specially when auto-BM cells were ex vivo loaded with the TNF transgene as compared to treatment with free vMyx-hTNF (Figure 2B).

Another important aspect of our study is that the means of systemic delivery of oncolytic MYXV matters because by using BM autographs ex vivo loaded with MYXV, superior therapeutic effects (i.e., 50\% overall survival) were observed as compared to the systemic delivery of the free virus, which was ineffective compared to the delivery using autologous BM armed with the virus (i.e., 10-20\% overall survival rates) (Figure $2 \mathrm{~B}$ ). In principle, the observed improvement of survival rates in this mouse model with established myeloma when treated with BM/MYXV can be attributed to multiple reasons: (i) an increase in the dosage of oncolytic MYXV delivered to the tumor microenvironment in the BM resulting in more effective oncolysis; (ii), MYXV together with the carrier BM cells, can trigger a native or adaptive immune response resulting in superior anti-tumor effects; or (iii) a combination of both (i) and (ii). In contrast, the systemic delivery of free virus can encounter physiological barriers such as virus neutralization by antibodies, antiviral cytokines and tissue-resident macrophages, resulting in a rapid clearance of the virus from the circulation before it reaches the cancerous target in the bone marrow. Also, the non-specific uptake of the virus by the spleen and/or liver, for further clearance [34], and the poor virus escape from the vascular compartment [35]. Also, even though only a small fraction of mice survived this regimen, all of the survivors became resistant to a second challenge with fresh Vk12598 cells (Figure 2B, 2C), suggesting that these re-challenged mice have become resistant against aggressive and drug refractory MM cells, and importantly these results suggest that the re-challenged mice have developed acquired anti-tumor immunity to the myeloma cells.

In the past, others have shown that the oncolytic reovirus synergizes with the standard-of-care MM drug bortezomib against MM using the Vk12598 murine model of MM [22]. This result was surprising considering that Vk12598 cells are insensitive to bortezomib (BOR) treatment. However, the authors found that BOR augmented reovirus replication in MM cells and in tumor-associated endothelial cells, increasing viral delivery and consequently stimulation of cytokine release, apoptosis, immune activity and reduction of anti-tumor immunosuppression [22]. Therefore, we hypothesize that combination therapy with MYXV and chemo/ immunotherapeutic agents might also increase survival and eradicate myeloma in vivo. To test this hypothesis, mice bearing MM tumors were treated with the alkylating agent $\mathrm{Cy}$ in combination with autologous $\mathrm{BM}$ ex vivo loaded with MYXV. As mentioned before, $\mathrm{Cy}$ is one of the forefront chemotherapeutic drugs used to treat multiple myeloma specially in combination with other chemotherapeutic agents [36]. Unfortunately, the 
anti-myeloma effects of this alkylating agent by itself are not sustained for a long period of time and eventually the disease relapses [36]. For our in vivo experiments, mice were seeded with myeloma cells for 3 weeks, which assures the establisment of later-stage disease. Then mice were treated with $\mathrm{Cy}$ twice, each dose 1 week apart in order to induce minimal residual disease (MRD). After this, mice were treated with auto-BM ex vivo loaded with unarmed vMyx-M135KO or with armed vMyxhTNF. As shown in Figure 3B, we could not observe any synergistic effect with this combination therapy. Although the progression of the disease was delayed, no statistically significant differences were observed among the cohorts treated with $\mathrm{Cy}$ alone (28\% overall survival) versus $\mathrm{Cy}$ followed by BM/ vMyx-M135KO (50\% overall survival) or BM/vMyx-hTNF (25\% overall survival). Nevertheless, the therapeutic benefits with treatment including $\mathrm{Cy}$ followed by BM/vMyx-M135KO were modestly superior than that one using $\mathrm{Cy}$ and BM/vMyx-hTNF, implying that the TNF transgene might have actually interfered with effacacy. One possible reason for this observation is that combination of Cy and MYXV armed with hTNF might have induced undesirable side effects, including cytotoxicity towards T lymphocytes. In addition, it has been reported that TNF along with IL-6, IL-1 $\beta$ synergizes with receptor activator of nuclear factor $\kappa \mathrm{B}$ ligand (RANKL) to induce osteoclastogenesis in MM [37, 38].

Finally, we explored combination therapy, including the LCL161 Smac-mimetics compound plus the ICI $\alpha$-PD1 followed by transplantation with BM leukocyte carrier cells armed with MYXV. The Smac-mimetics compound LCL161 completed a phase 1-2 clinical trials for MM. This compound acts by debulking human osteoclasts and compromising their viability [39]. On the other hand, the use of $\alpha-P D-1$ as monotherapy against MM has demonstrated lack of activity in clinical trials [40]. In 2016, Chesi and co-workers demonstrated outstanding therapeutic benefits with the combination of LCL161 plus $\alpha$-PD1 in the transplantable Vk*MYC murine MM model [12]. In fact, the authors demonstrated synergistic effects of this combination therapy and acquisition of immunity by survivor mice that were re-challenged with $\mathrm{Vk} * \mathrm{MYC}$ cells. We noted dramatic M-Spike reductions in the LCL161/ $\alpha$ PD1 cohort by 25 days (Figure 4C) and we did observe that the combination of LCL161/ $\alpha$-PD-1 followed by autologous transplantation with $\mathrm{BM}$ cells ex vivo loaded with either vMyx-M135KO or vMyx-hTNF, had additional therapeutic benefits in mice seeded with Vk12598 cells (i.e., overall survival with vMyx-M135KO $=75 \%$ vs. vMyx-hTNF $=$ 60\%) (Figure 4). Furthermore, results shown in Figure 5 suggest that survivor mice might have acquired immunity against re-challenging with Vk12598 cells.

Together, we show promising results in terms of therapeutic benefits of delivering oncolytic MYXV via carrier cells from autologous BM transplants, both alone or in combination with LCL161 and $\alpha$-PD-1 against drug-resistant $\mathrm{MM}$ cells in vivo. To our knowledge, these are the first results showing therapeutic benefits of oncolytic MYXV to control and even eradicate established drug-resistant MM cells in a preclinical murine model that has previously shown excellent concordance with predicting clinical efficacy in human MM patients. Although in this study, we have demonstrated that autologous bone marrow leukocytes can efficiently be packed with an oncolytic virus like MYXV, resulting in therapeutic effects against drug resistant MM cells, future studies should be focused on understanding the mechanisms by which oncolytic virotherapy with MYXV alone or in combination with chemotherapeutic/ immunotheraputic agents results in cancer-free or cancercontrolling outcomes in the pre-clinical murine $\mathrm{Vk}^{*} \mathrm{MYC}$ model. In the past, using a different MM murine model we have also demonstrated that certain autologous or allogeneic bone marrow or PBMCs derived leukocytes are better carriers of MYXV into the tumor microenvironment of MM [27, 28]. Therefore, it will be important to identify potential autologous BM leukocyte carriers of MYXV using the $\mathrm{Vk}^{*} \mathrm{MYC}$ model.

\section{MATERIALS AND METHODS}

\section{Cells and viruses}

The murine bortezomib resistant Vk12598 was a kind gift of Drs. Marta Chesi (Ph.D.), and P. Leif Bergsagel (M.D.) (Mayo Clinic, Arizona). These cells were used to perform in vitro and in vivo assays.

Three myxoma virus constructs were used in this study: vMyx-M093L-Venus (wild-type MYXV that expresses Venus-tagged M093 protein as a virion component), vMyx-M135KO (an un-armed and attenuated recombinant MYXV, in which the M135 gene has been deleted and the green fluorescent protein (GFP) has been inserted under a poxvirus synthetic early/late promoter) [41] and vMyx-hTNF (GFP-expressing knock in of the human TNF gene inserted into the M131 gene) [13, 41, 42].

\section{Myxoma virus binding and infection of $\mathrm{Vk12598}$ myeloma cells}

For virus binding, Vk12598 MM cells were incubated with vMyx-M093L-Venus at an MOI $=10$ for 1 hour at $4{ }^{\circ} \mathrm{C}$ to allow virus particles binding to cells. Unbound virus was washed twice with 1x-PBS supplemented with 5\% FBS. For virus infection, vMyxM135KO expressing the GFP protein driven by an early/late viral promoter was incubated at $\mathrm{MOI}=10$ with Vk12598 cells for 1 hour at $37^{\circ} \mathrm{C}$ to allow virus adsorption. After these, cells were incubated at $37^{\circ} \mathrm{C}$ for 18 hours to allow virus infection. Virus binding to the Vk12598 MM (CD138 $\left.{ }^{+} \mathrm{CD}_{22}{ }^{-}\right)$cells or virus infection of these myeloma cells was assessed using flow cytometry. 


\section{Flow cytometry analysis}

To isolate spleen and BM, mice were euthanized by $\mathrm{CO}_{2}$ inhalation. Once collected, from the spleen a singlecell suspension was obtained by mechanical disruption between frosted glass slides. On the other hand, BM cells were collected by flushing out ilium, femur, tibia, humerus, and radius with 1x-PBS. On average, about $1.0 \times 10^{8}$ of BM nucleated cells were obtained from each mouse. These samples were used to perform virus binding and infection assays, as well as to determine the number of MM cells remaining after virus infection. For this latter assay, Vk12598 (CD138 ${ }^{+}$) cells mock treated (i.e., without adding the virus) or infected with vMy-M135KO were labeled with near-IR-live/dead stain (Invitrogen). To assess of percentage of $\mathrm{MM}$ cells $\left(\mathrm{B} 220^{-} \mathrm{CD} 138^{+}\right)$in bone marrow and spleen and to quantify levels of virus binding and infection of $\mathrm{CD} 138^{+} \mathrm{CD} 220^{-} \mathrm{MM}$ cells and the cell viability upon virus infection of these myeloma cells flow cytometry was used according to described protocols [9]. Fluorescently labeled monoclonal antibodies such as B220-APC (clone RA3-6B2) and CD138-BV605 (clone 281-2) were from Biolegend. For each experimental condition a minimum of 300,000 events were acquired on a LSRFortessa cytometer (BD Biosciences) and acquired data were analyzed using the FlowJo software.

\section{Administration of chemotherapeutic drugs and $\alpha$-PD-1 check point inhibitor}

For drug dosage and route of administration, we followed reported methods [12]. In brief, cyclophosphamide (Cy) (Tocris Bioscience) was used at a final concentration of $100 \mathrm{mg} / \mathrm{Kg}$ and administrated intraperitoneally (i.p.) twice, each dose one week apart. When LCL161 (Chemietek) was used, $50 \mathrm{mg} / \mathrm{Kg}$ LCL161 was administrated via the oral gavage (o.g.) route. The checkpoint inhibitor $\alpha$-PD-1 (BioXCell) was administrated intraperitoneally (i.p.) at a final concentration of $10 \mathrm{mg} /$ $\mathrm{Kg}$. One week after cancer implantation, both LCL161 and $\alpha$-PD-1 were administrated on days $1,4,8,11$ as described in Figure 4A.

\section{In vivo mouse studies}

All animal experiments were performed under the Institutional Animal Care and Use Committee (IACUC) approval (no. 171543R) of Arizona State University and conformed to all regulatory standards. For in vivo autologous BM-derived leukocyte transplantations, we used, 8-weeks old C57BL/6 female or male mice, (Charles River laboratories, Wilmington, MA) as donors or recipients, which were matched according to gender and age. First, immunocompetent $\mathrm{C} 57 \mathrm{BL} / 6$ recipient mice were implanted intravenously (i.v.) via tail vein with $1.0 \times 10^{6}$ BOR-resistant Vk12598 cells. One week after myeloma implantation, mice were randomized to six groups or cohorts (i.e., a minimum of 7 or 10 mice per group, unless other number specified) and treated as follows: No BM transplant but 1x-PBS vehicle control (cohort I); $2.0 \times 10^{6}$ autologous BM cells alone (cohort II); $1.0 \times 10^{7}$ focus forming (i.e. infectious) units (ffu) of free un-armed vMyx-M135KO virus construct (cohort III); $2.0 \times 10^{6} \mathrm{BM}$ cells ex vivo pre-loaded with un-armed vMyx-M135KO at a multiplicity of infection (MOI) of 10 , (cohort IV); $1 \times 10^{7} \mathrm{ffu}$ of free transgene-armed vMyxhTNF virus construct (cohort V); $2.0 \times 10^{6}$ autologous $\mathrm{BM}$ cells ex vivo treated with transgene-armed vMyxhTNF virus construct at MOI of 10 (cohort VI). Each treatment was delivered via retro-orbital (r.o.) route. At the onset of morbidity (i.e., hind limb paralysis, hunched position, weight loss, labor breathing, or at the end of the study, mice were euthanized via asphyxiation with $\mathrm{CO}_{2}$, as per institutional guidelines. Survival was assessed 100 days following cancer implantation. For re-challenge experiments, MYXV-treated mice from transplant cohorts that survived 100 days, post-cancer implantation were reinfused with fresh $1.0 \times 10^{6} \mathrm{Vk} 12598$ cells intravenously (i.v.) via tail vein. Mice that survived more than 100 days post cancer re-challenged were considered cured and had acquired immunity to the myeloma.

For in vivo experiments using the chemotherapeutic compound cyclophosphamide (Cy), mice were injected with $1 \times 10^{6} \mathrm{Vk} 12598$ cells and the disease was allowed to be established for 3 weeks. Then mice were treated twice with Cy in order to debulk the tumor burden. It is known that $\mathrm{Cy}$ induces a transient anti-myeloma response [36]. Then mice were treated as follows: $1 \mathrm{x}-\mathrm{PBS}$, vehicle control (cohort I), cyclophosphamide alone (cohort II), Cy followed by treatment with $2.0 \times 10^{6}$ of autologous BM cells ex vivo loaded with un-armed vMyx-M135KO at a multiplicity of infection (MOI) of 10, (cohort III), or 2.0 $\times 10^{6}$ of autologous BM cells ex-vivo loaded with vMyxhTNF at MOI of 10 (cohort IV). Likewise, combination therapy with $50 \mathrm{mg} / \mathrm{Kg} \mathrm{LCL} 161$ and $10 \mathrm{mg} / \mathrm{Kg} \alpha-\mathrm{PD}-$ 1 , as described before, was followed by autologous BM transplantation + MYXV. In brief mice were treated as follows: 1x-PBS (cohort I); LCL161 $+\alpha$-PD-1 (cohort II); LCL161 + $\alpha$-PD-1 + free vMyx-M135KO (cohort III); LCL161 + $\alpha$-PD-1 + free vMyx-hTNF (cohort IV); LCL161 + $\alpha$-PD-1 + BM + vMyx-M135KO (cohort V); LCL161 + $\alpha$-PD-1 + free vMyx-hTNF (cohort VI).

\section{Assessment of myeloma burden using serum protein electrophoresis (SPEP)}

Blood was collected from mice periodically by cheek bleeding. About $100 \mu \mathrm{L}$ of whole blood were collected into microtainer tubes (BD Biosciences), allowed to coagulate at room temperature, and spun for 10 minutes at 2,300 $\times g$. Sera were diluted 1:2 in normal saline buffer and analyzed on a QuickGel Chamber apparatus using 
pre-casted QuickGels (Helena Laboratories) according to the manufacturer's instruction. Densitometric analysis was then performed using the clinically certified Helena QuickScan 2000 workstation which allows a precise quantization of the various serum fractions, including the measurements of gamma/albumin $(\mathrm{G} / \mathrm{A})$ ratio. A G/A ratio between $0.5-2.0$ corresponds to predominant $\mathrm{M}$-spikes and suggest not only good multiple myeloma engraftment but also tumor response to treatments in similar factions as is done clinically [10].

\section{Statistics}

Values are represented as means \pm SD for at least two or three independent experiments.

Kaplan-Meier analysis of mouse survival was performed with GraphPad Prism 8 software (La Jolla, CA, USA), and log-rank (Mantel-Cox) test was performed to compare survival curves and to perform statistical analysis. Statistical comparison between two groups was conducted using the 2-tailed Student's $t$-test. Animals were assigned to treatment groups (Cohorts) randomly and the number of animals in each treatment group is reported in the figures. $P$ values are reported as follows: no significant (ns) $P>0.05,{ }^{*} P<0.05,{ }^{* *} P 0.01,{ }^{* * *} P 0.001,{ }^{* * * *} P 0.0001$.

\section{Study approval}

All animal work was conducted under the approval of Arizona State University Institutional Animal Care and Use Committee (IACUC) (no. 171543R) in accordance with federal, state, and local guidelines.

\section{Author contributions}

N.Y.V. designed and performed experiments, analyzed data and wrote the manuscript. M.M.R. performed experiments and analyzed data. J.M., M.E.S., J.K., K.L., J.D-V., J.D., E.G., performed experiments. M.C. and L.B. provided the murine Vk12598 myeloma cells and facilitated the performing of the SPEP experiments. G.M. provided overall project leadership, supervised the data analysis. All authors reviewed the manuscript.

\section{ACKNOWLEDGMENTS}

We want to thank Drs. Marta Chesi (Ph.D) and P. Leif Bergsagel (MD) from Mayo Clinic, Arizona for providing the VK12598 cell line and for their valuable scientific comments and suggestions.

\section{CONFLICTS OF INTEREST}

Grant McFadden (GM) is co-founder and equity holder of OncoMyx Therapeutics. M.M.R. is a consultant for OncoMyx Therapeutics.

\section{FUNDING}

This work was funded by an Arizona State University (ASU) start-up grant to GM, NIAID R01 AI080607 to MMR and GM, Mayo Clinic Developmental Research Award from the NCI Myeloma SPORE grant (GR35414), awarded to PLB.

\section{REFERENCES}

1. Rajkumar SV. Multiple myeloma: Every year a new standard? Hematol Oncol. 2019; 37:62-65. https://doi. org/10.1002/hon.2586. [PubMed]

2. Galson DL, Silbermann R, Roodman GD. Mechanisms of multiple myeloma bone disease. Bonekey Rep. 2012; 1:135. https://doi.org/10.1038/bonekey.2012.135. [PubMed]

3. Mehta A. Multiple myeloma. Hematology. 2015; 20:5859. https://doi.org/10.1179/1024533214Z.000000000333. [PubMed]

4. Rossi M, Botta C, Arbitrio M, Grembiale RD, Tagliaferri P, Tassone P. Mouse models of multiple myeloma: technologic platforms and perspectives. Oncotarget. 2018; 9:20119-33. https://doi.org/10.18632/oncotarget.24614. [PubMed]

5. Rajkumar SV, Kumar S. Multiple Myeloma: Diagnosis and Treatment. Mayo Clin Proc. 2016; 91:101-19. https://doi. org/10.1016/i.mayocp.2015.11.007. [PubMed]

6. Lilly CL, Villa NY, Lemos de Matos A, Ali HM, Dhillon JS, Hofland T, Rahman MM, Chan W, Bogen B, Cogle C, McFadden G. Ex Vivo Oncolytic Virotherapy with Myxoma Virus Arms Multiple Allogeneic Bone Marrow Transplant Leukocytes to Enhance Graft versus Tumor. Mol Ther Oncolytics. 2017; 4:31-40. https://doi.org/10.1016/j. omto.2016.12.002. [PubMed]

7. Villa NY, Rahman MM, Mamola J, D'Isabella J, Goras E, Kilbourne J, Lowe K, Daggett-Vondras J, Torres L, Christie J, Appel N, Cox AL, Kim JB, McFadden G. Autologous Transplantation Using Donor Leukocytes Loaded Ex Vivo with Oncolytic Myxoma Virus Can Eliminate Residual Multiple Myeloma. Mol Ther Oncolytics. 2020; 18:171-88. https://doi.org/10.1016/j. omto.2020.06.011. [PubMed]

8. Hofgaard PO, Jodal HC, Bommert K, Huard B, Caers J, Carlsen H, Schwarzer R, Schünemann N, Jundt F, Lindeberg MM, Bogen B. A novel mouse model for multiple myeloma (MOPC315.BM) that allows noninvasive spatiotemporal detection of osteolytic disease. PLoS One. 2012; 7:e51892. https://doi.org/10.1371/journal. pone.0051892. [PubMed]

9. Chesi M, Robbiani DF, Sebag M, Chng WJ, Affer M, Tiedemann R, Valdez R, Palmer SE, Haas SS, Stewart AK, Fonseca R, Kremer R, Cattoretti G, Bergsagel PL. AIDdependent activation of a MYC transgene induces multiple myeloma in a conditional mouse model of post-germinal center malignancies. Cancer Cell. 2008; 13:167-80. https:// doi.org/10.1016/j.ccr.2008.01.007. [PubMed] 
10. Chesi M, Matthews GM, Garbitt VM, Palmer SE, Shortt J, Lefebure M, Stewart AK, Johnstone RW, Bergsagel PL. Drug response in a genetically engineered mouse model of multiple myeloma is predictive of clinical efficacy. Blood. 2012; 120:376-85. https://doi.org/10.1182/ blood-2012-02-412783. [PubMed]

11. Steiner N, Nachbaur D, König $P$, Willenbacher $W$, Gunsilius E. Long-term control in a patient with refractory multiple myeloma by oral cyclophosphamide and dexamethasone. Anticancer Res. 2015; 35:2165-68. [PubMed]

12. Chesi M, Mirza NN, Garbitt VM, Sharik ME, Dueck AC, Asmann YW, Akhmetzyanova I, Kosiorek HE, Calcinotto A, Riggs DL, Keane N, Ahmann GJ, Morrison KM, et al. IAP antagonists induce anti-tumor immunity in multiple myeloma. Nat Med. 2016; 22:1411-20. https://doi. org/10.1038/nm.4229. [PubMed]

13. Christie JD, Appel N, Canter H, Achi JG, Elliott NM, de Matos AL, Franco L, Kilbourne J, Lowe K, Rahman MM, Villa NY, Carmen J, Luna E, et al. Systemic delivery of TNF-armed myxoma virus plus immune checkpoint inhibitor eliminates lung metastatic mouse osteosarcoma. Mol Ther Oncolytics. 2021; 22:539-54. https://doi. org/10.1016/j.omto.2021.07.014. [PubMed]

14. Ghiringhelli F, Larmonier N, Schmitt E, Parcellier A, Cathelin D, Garrido C, Chauffert B, Solary E, Bonnotte B, Martin F. CD4+CD25+ regulatory T cells suppress tumor immunity but are sensitive to cyclophosphamide which allows immunotherapy of established tumors to be curative. Eur J Immunol. 2004; 34:336-44. https://doi.org/10.1002/ eji.200324181. [PubMed]

15. Ghiringhelli F, Menard C, Puig PE, Ladoire S, Roux S, Martin F, Solary E, Le Cesne A, Zitvogel L, Chauffert B. Metronomic cyclophosphamide regimen selectively depletes $\mathrm{CD} 4+\mathrm{CD} 25+$ regulatory $\mathrm{T}$ cells and restores $\mathrm{T}$ and NK effector functions in end stage cancer patients. Cancer Immunol Immunother. 2007; 56:641-48. https:// doi.org/10.1007/s00262-006-0225-8. [PubMed]

16. Lutsiak ME, Semnani RT, De Pascalis R, Kashmiri SV, Schlom J, Sabzevari H. Inhibition of CD4(+)25+ T regulatory cell function implicated in enhanced immune response by low-dose cyclophosphamide. Blood. 2005; 105:2862-68. https://doi.org/10.1182/blood-2004-06-2410. [PubMed]

17. Meermeier EW, Welsh SJ, Sharik ME, Du MT, Garbitt VM, Riggs DL, Shi CX, Stein CK, Bergsagel M, Chau B, Wheeler ML, Bezman N, Wang F, et al. Tumor burden limits bispecific antibody efficacy through $\mathrm{T}$ cell exhaustion averted by concurrent cytotoxic therapy. Blood Cancer Discov. 2021; 2:354-69. https://doi.org/10.1158/2643-3230. BCD-21-0038. [PubMed]

18. Srivastava S, Furlan SN, Jaeger-Ruckstuhl CA, Sarvothama M, Berger C, Smythe KS, Garrison SM, Specht JM, Lee SM, Amezquita RA, Voillet V, Muhunthan V, YechanGunja S, et al. Immunogenic Chemotherapy Enhances
Recruitment of CAR-T Cells to Lung Tumors and Improves Antitumor Efficacy when Combined with Checkpoint Blockade. Cancer Cell. 2021; 39:193-208.e10. https://doi. org/10.1016/j.ccell.2020.11.005. [PubMed]

19. Kambara H, Saeki Y, Chiocca EA. Cyclophosphamide allows for in vivo dose reduction of a potent oncolytic virus. Cancer Res. 2005; 65:11255-58. https://doi. org/10.1158/0008-5472.CAN-05-2278. [PubMed]

20. Kirn D. Clinical research results with d11520 (Onyx-015), a replication-selective adenovirus for the treatment of cancer: what have we learned? Gene Ther. 2001; 8:89-98. https:// doi.org/10.1038/sj.gt.3301377. [PubMed]

21. Infante JR, Dees EC, Olszanski AJ, Dhuria SV, Sen S, Cameron S, Cohen RB. Phase I dose-escalation study of LCL161, an oral inhibitor of apoptosis proteins inhibitor, in patients with advanced solid tumors. J Clin Oncol. 2014; 32:3103-10. https://doi.org/10.1200/JCO.2013.52.3993. [PubMed]

22. Thirukkumaran CM, Shi ZQ, Nuovo GJ, Luider J, Kopciuk KA, Dong Y, Mostafa AA, Thakur S, Gratton K, Yang A, Chin AC, Coffey MC, Jimenez-Zepeda VH, et al. Oncolytic immunotherapy and bortezomib synergy improves survival of refractory multiple myeloma in a preclinical model. Blood Adv. 2019; 3:797-812. https://doi.org/10.1182/ bloodadvances.2018025593. [PubMed]

23. Packiriswamy N, Upreti D, Zhou Y, Khan R, Miller A, Diaz RM, Rooney CM, Dispenzieri A, Peng KW, Russell SJ. Oncolytic measles virus therapy enhances tumor antigenspecific T-cell responses in patients with multiple myeloma. Leukemia. 2020; 34:3310-22. https://doi.org/10.1038/ s41375-020-0828-7. [PubMed]

24. Lei W, Wang S, Xu N, Chen Y, Wu G, Zhang A, Chen X, Tong Y, Qian W. Enhancing therapeutic efficacy of oncolytic vaccinia virus armed with Beclin-1, an autophagic Gene in leukemia and myeloma. Biomed Pharmacother. 2020; 125:110030. https://doi.org/10.1016/j.biopha.2020.110030. [PubMed]

25. Calton CM, Kelly KR, Anwer F, Carew JS, Nawrocki ST. Oncolytic Viruses for Multiple Myeloma Therapy. Cancers (Basel). 2018; 10:198. https://doi.org/10.3390/ cancers 10060198. [PubMed]

26. Thirukkumaran CM, Luider JM, Stewart DA, Cheng T, Lupichuk SM, Nodwell MJ, Russell JA, Auer IA, Morris DG. Reovirus oncolysis as a novel purging strategy for autologous stem cell transplantation. Blood. 2003; 102:377-87. https://doi.org/10.1182/blood-2002-08-2508. [PubMed]

27. Russell SJ, Peng KW, Bell JC. Oncolytic virotherapy. Nat Biotechnol. 2012; 30:658-70. https://doi.org/10.1038/ $\underline{\text { nbt.2287. }}$ [PubMed]

28. Sivanandam V, LaRocca CJ, Chen NG, Fong Y, Warner SG. Oncolytic Viruses and Immune Checkpoint Inhibition: The Best of Both Worlds. Mol Ther Oncolytics. 2019; 13:93-106. https://doi.org/10.1016/j.omto.2019.04.003. [PubMed] 
29. Rahman MM, McFadden G. Oncolytic Viruses: Newest Frontier for Cancer Immunotherapy. Cancers (Basel). 2021; 13:5452. https://doi.org/10.3390/cancers 13215452. [PubMed]

30. Olechnowicz SWZ, Weivoda MM, Lwin ST, Leung SK, Gooding S, Nador G, Javaid MK, Ramasamy K, Rao SR, Edwards JR, Edwards CM. Multiple myeloma increases nerve growth factor and other pain-related markers through interactions with the bone microenvironment. Sci Rep. 2019; 9:14189. https://doi.org/10.1038/s41598-019-505915. [ PubMed]

31. Hideshima T, Chauhan D, Schlossman R, Richardson P, Anderson KC. The role of tumor necrosis factor alpha in the pathophysiology of human multiple myeloma: therapeutic applications. Oncogene. 2001; 20:4519-27. https://doi. org/10.1038/sj.onc.1204623. [PubMed]

32. Marino S, Roodman GD. Multiple Myeloma and Bone: The Fatal Interaction. Cold Spring Harb Perspect Med. 2018; 8:a031286. https://doi.org/10.1101/cshperspect.a031286. [PubMed]

33. Li Y, Lin Y. Tumor Necrosis Factor Alpha-308G/A Polymorphism and the Risk of Multiple Myeloma: A MetaAnalysis of Pooled Data from Twelve Case-Control Studies. Turk J Haematol. 2019; 36:72-80. https://doi.org/10.4274/ tjh.galenos.2019.2018.0238. [PubMed]

34. Ferguson MS, Lemoine NR, Wang Y. Systemic delivery of oncolytic viruses: hopes and hurdles. Adv Virol. 2012; 2012:805629. https://doi.org/10.1155/2012/805629. [PubMed]

35. Wong HH, Lemoine NR, Wang Y. Oncolytic Viruses for Cancer Therapy: Overcoming the Obstacles. Viruses. 2010; 2:78-106. https://doi.org/10.3390/v2010078. [PubMed]
36. Schjesvold F, Oriol A. Current and Novel Alkylators in Multiple Myeloma. Cancers (Basel). 2021; 13:2465. https:// doi.org/10.3390/cancers13102465. [PubMed]

37. Teitelbaum SL. Bone resorption by osteoclasts. Science. 2000; 289:1504-8. https://doi.org/10.1126/ science.289.5484.1504. [PubMed]

38. Del Fattore A, Teti A, Rucci N. Osteoclast receptors and signaling. Arch Biochem Biophys. 2008; 473:147-60. https://doi.org/10.1016/j.abb.2008.01.011. [ubMed]

39. Moen IN, Westhrin M, Håland E, Haug M, Nonstad U, Klaharn M, Standal T, Starheim KK. Smac-mimetics reduce numbers and viability of human osteoclasts. Cell Death Discov. 2021; 7:36. https://doi.org/10.1038/s41420-02100415-1. [PubMed]

40. Jelinek T, Paiva B, Hajek R. Update on PD-1/PD-L1 Inhibitors in Multiple Myeloma. Front Immunol. 2018; 9:2431. $\quad$ https://doi.org/10.3389/fimmu.2018.02431. [PubMed]

41. Barrett JW, Sypula J, Wang F, Alston LR, Shao Z, Gao X, Irvine TS, McFadden G. M135R is a novel cell surface virulence factor of myxoma virus. J Virol. 2007; 81:106-14. https://doi.org/10.1128/JVI.01633-06. [PubMed]

42. Lun X, Yang W, Alain T, Shi ZQ, Muzik H, Barrett JW, McFadden G, Bell J, Hamilton MG, Senger DL, Forsyth PA. Myxoma virus is a novel oncolytic virus with significant antitumor activity against experimental human gliomas. Cancer Res. 2005; 65:9982-90. https://doi. org/10.1158/0008-5472.CAN-05-1201. [PubMed] 